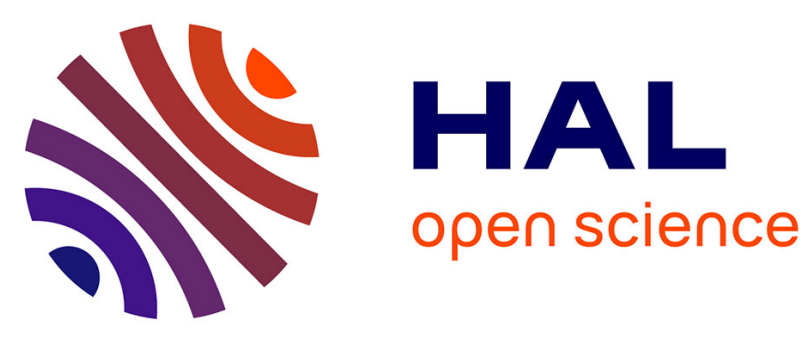

\title{
A Multiscale Model Based On Intragranular Microstructure: Influence Of Grain-Scale Substructure On Macroscopic Behaviour Of An IF-Steel During Complex Load Paths
}

Gérald Franz, Farid Abed-Meraim, Tarak Ben Zineb, Xavier Lemoine, Marcel Berveiller

\section{To cite this version:}

Gérald Franz, Farid Abed-Meraim, Tarak Ben Zineb, Xavier Lemoine, Marcel Berveiller. A Multiscale Model Based On Intragranular Microstructure: Influence Of Grain-Scale Substructure On Macroscopic Behaviour Of An IF-Steel During Complex Load Paths. NUMIFORM 07: Materials Processing and Design: Modeling, Simulation and Applications, Jun 2007, Porto, Portugal. pp.13451350, 10.1063/1.2740996 . hal-01206835

\section{HAL Id: hal-01206835 \\ https://hal.science/hal-01206835}

Submitted on 29 Sep 2015

HAL is a multi-disciplinary open access archive for the deposit and dissemination of scientific research documents, whether they are published or not. The documents may come from teaching and research institutions in France or abroad, or from public or private research centers.
L'archive ouverte pluridisciplinaire HAL, est destinée au dépôt et à la diffusion de documents scientifiques de niveau recherche, publiés ou non, émanant des établissements d'enseignement et de recherche français ou étrangers, des laboratoires publics ou privés. 


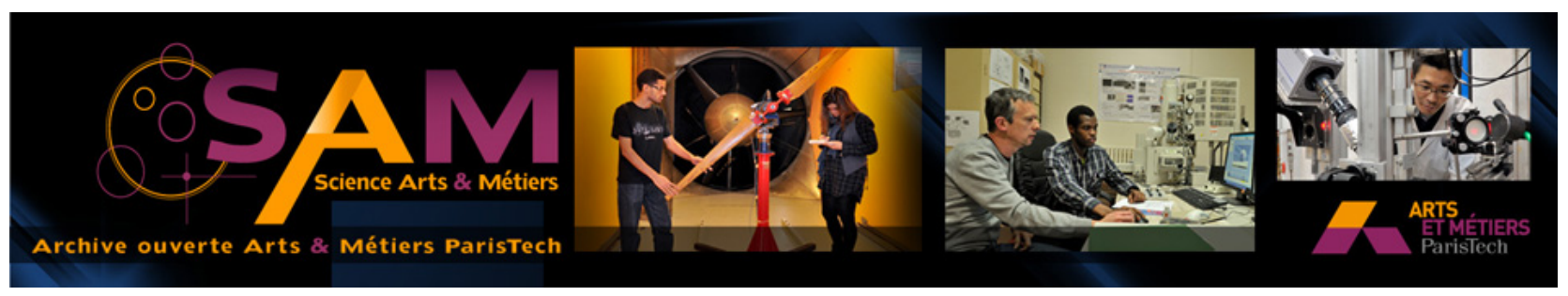

\section{Science Arts \& Métiers (SAM)}

is an open access repository that collects the work of Arts et Métiers ParisTech researchers and makes it freely available over the web where possible.

This is an author-deposited version published in: http://sam.ensam.eu

Handle ID: .http://hdl.handle.net/10985/10249

\section{To cite this version :}

Gérald FRANZ, Farid ABED-MERAIM, Tarak BEN ZINEB, Xavier LEMOINE, Marcel

BERVEILLER - A Multiscale Model Based On Intragranular Microstructure: Influence Of Grain-

Scale Substructure On Macroscopic Behaviour Of An IF-Steel During Complex Load Paths - In:

NUMIFORM 07: Materials Processing and Design: Modeling, Simulation and Applications,

Portugal, 2007-06 - AIP Conference Proceedings: Numiform'07 - 2007 


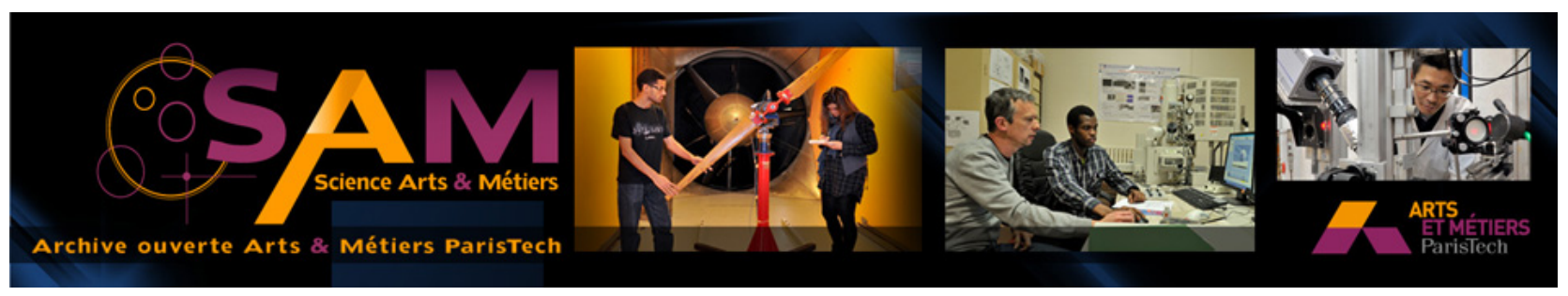

\section{Science Arts \& Métiers (SAM)}

is an open access repository that collects the work of Arts et Métiers ParisTech researchers and makes it freely available over the web where possible.

This is an author-deposited version published in: http://sam.ensam.eu

Handle ID: .http://hdl.handle.net/null

\section{To cite this version :}

Gérald FRANZ, Farid ABED-MERAIM, Tarak BEN ZINEB, Xavier LEMOINE, Marcel

BERVEILLER - A Multiscale Model Based On Intragranular Microstructure: Influence Of Grain-

Scale Substructure On Macroscopic Behaviour Of An IF-Steel During Complex Load Paths - In:

NUMIFORM 07: Materials Processing and Design: Modeling, Simulation and Applications,

Portugal, 2007-06 - AIP Conference Proceedings: Numiform'07 - 2007 


\title{
A Multiscale Model Based On Intragranular Microstructure: Influence Of Grain-Scale Substructure On Macroscopic Behaviour Of An IF-Steel During Complex Load Paths
}

\author{
Gérald Franz ${ }^{1}$, Farid Abed-Meraim ${ }^{1}$, Tarak Ben Zineb ${ }^{2}$, \\ Xavier Lemoine ${ }^{3}$, Marcel Berveiller ${ }^{1}$ \\ 1 LPMM, UMR CNRS 7554, ENSAM 4 rue Augustin Fresnel, 57078 Metz Cedex 3, France \\ 2 LEMTA, UMR CNRS 7563, Nancy Université 2 rue Jean Lamour, 54519 Vandouvre-lès-Nancy, France \\ 3 Centre Automobile Produit, Arcelor Research S.A., voie Romaine B.P. 30320, 57283 Maizières-lès-Metz, France
}

\begin{abstract}
A microstructural model, based on Peeters' works, is implemented into a large strain self-consistent scheme, leading to the multiscale model which achieves, for each grain, the calculation of slip activity, with help of regularized formulation drawn from the visco-plasticity framework, and the dislocation microstructure evolution. This paper focuses on the relationship between macroscopic hardening/softening effects and induced microstructure during monotonic and two-stage strain paths.
\end{abstract}

Keywords: Crystal plasticity, Microstructure, Dislocations, Complex strain paths.

PACS: $61.72 . F f, 61.72 .-y, 62.20 . F e, ~ 83.10 . G r, 83.60 .-\mathrm{a}$

\section{INTRODUCTION}

The development of a relevant constitutive model adatped to sheet metal forming simulations requires an accurate description of the most important sources of anisotropy, i.e. the slip processes, the intragranular substructure changes and the texture development. During plastic deformation of thin metallic sheets, strain-path changes often occur in the material resulting in macroscopic effects. These softening/hardening effects must be correctly predicted because they can significantly influence the strain distribution and may lead to flow localization and material failure. The main origin of these effects is related to the intragranular microstructure evolution. This implies that an accurate description of the dislocation patterning during monotonic or complex strain-paths is needed to lead to a reliable constitutive model.

This paper focuses on the modelling of collective dislocation processes. The substructure is described taking account of the experimental observations as stress-strain curves and TEM micrographs. Three local dislocation densities, introduced as internal variables in the multiscale model, allow representing the spatially heterogeneous distributions of dislocations inside grains. Rate equations, based on the consideration of associated creation, storage and annihilation, are adopted to describe the dislocation cells evolution. The coupling of the substructure to the critical shear stresses is performed thanks to the concepts of isotropic hardening, latent hardening and polarity.

In this paper, a crystal plasticity model coupled with a description of the intragranular microstructural evolution is presented. Five mechanical tests, namely a monotonic loading path, two cross tests and two reverse tests, are sufficient to identify the model parameters. It is shown that each parameter contributes mainly to one feature in the macroscopic plastic material behaviour, without influencing significantly the other softening/hardening effects. Moreover, it is also shown that the model successfully reproduces the dislocation substructures observed in TEM after monotonic deformation, reverse tests and cross tests for crystal with stable and instable initial orientation. It seems that the rotation of the lattice orientation influences on the Bauschinger effect and the transient zone during reverse test.

\section{SINGLE CRYSTAL MODEL}

The elastic-plastic single crystal constitutive law written within the large strain framework presented 
here is developed in several works [1-5]. The single crystal behaviour is assumed to be elastic-plastic and the plastic deformation is only due to crystallographic slip. The other plastic deformation modes such as twinning or phase transformation are not considered. In BCC metals, 24 independent slip systems are assumed potentially active, i.e. the slip planes $\{110\}$ and $\{112\}$ and the slip directions $<111>$.

The strain rate of crystalline network (symmetric part) $d$ and the total spin (skew symmetric part) $w$ of the velocity gradient $g=d+w$ are related to elastic strain rate $d^{e}$ and slip rate $\dot{\gamma}$ by:

$$
\begin{aligned}
& d^{p}=d-d^{e}=R^{g} \dot{\gamma}^{g} \\
& w^{p}=w-w^{e}=S^{g} \dot{\gamma}^{g}
\end{aligned}
$$

where $R$ and $S$ are the symmetric and skew symmetric parts of the Schmid's tensor.

A slip system $g$ is active if its resolved shear stress $\tau^{g}$ reaches a critical value $\tau_{c}^{g}$ and if its rate $\dot{\tau}^{g}$ equals the critical shear stress rate $\dot{\tau}_{c}^{g}$. Consequently, the slip rate of the considered system is different from zero, so that:

$$
\begin{aligned}
& \tau^{g}<\tau_{c}^{g} \Rightarrow \dot{\gamma}^{g}=0 \\
& \tau^{g}=\tau_{c}^{g}, \dot{\tau}^{g}<\dot{\tau}_{c}^{g} \Rightarrow \dot{\gamma}^{g}=0 \\
& \tau^{g}=\tau_{c}^{g}, \dot{\tau}^{g}=\dot{\tau}_{c}^{g} \Rightarrow \dot{\gamma}^{g} \neq 0
\end{aligned}
$$

The relationship (2) will be expressed by a viscoplastic type regularization $\dot{\gamma}^{g}=k^{g} \dot{\tau}^{g}$, without reproducing any time-dependency, in order to avoid combination analysis and to save computing time. The new formulation is written for a single system $\mathrm{g}$ :

$$
k^{g}=k_{0}\left\{\frac{1}{2}\left(1+\tanh \left(k_{1}\left(\frac{\left|\tau^{g}\right|}{\tau_{c}^{g}}-1\right)\right)\right)\right\}\left\{\left\{\frac{1}{2}\left(1+\tanh \left(k_{2} \dot{\tau}^{g} \tau^{g}\right)\right)\right\}\right.
$$

where $k_{0}$ is inversely proportional to the self-hardening term.

In large strain framework, the elastic law is written as:

$$
\hat{\sigma}=C:\left(d-d^{p}\right)-\operatorname{\sigma race}(d)
$$

where $C$ is the four order elastic tensor and $\hat{\sigma}$ is the Cauchy stress co-rotational derivative. After some mathematical developments, the slip rate expression becomes:

$$
\dot{\gamma}^{g}=M^{g h} k^{h} R^{h}:(C-\sigma \otimes \delta): d
$$

where $\delta$ is the Kronecker's symbol and $M^{g h}=\left(\delta_{h g}+k^{h} R^{h}: C: R^{g}\right)^{-1}$.
The constitutive law is described using a tangent operator $l$ linking the nominal stress rate $\dot{n}=l: g$ with the velocity gradient $g$.

The nominal stress rate $\dot{n}$ is obtained starting from the Cauchy stress one $\dot{\sigma}$ :

$$
\dot{n}=\hat{\sigma}-w^{p} . \sigma+\sigma \cdot w^{p}-d . \sigma-\sigma \cdot w+\sigma \text { trace }(d)(6)
$$

Combining the previous equations, the single crystal incremental constitutive law is obtained:

$$
\begin{aligned}
& l_{i j k l}=\left[C_{i j k l}-\frac{1}{2}\left(\delta_{i k} \sigma_{l j}+\delta_{i l} \sigma_{k j}\right)-\frac{1}{2}\left(\sigma_{i k} \delta_{l j}-\sigma_{i l} \delta_{j k}\right)\right]- \\
& {\left[\left(C_{i j p q} R_{p q}^{g}+S_{i p}^{g} \sigma_{p j}-\sigma_{i p} S_{p j}^{g}\right) M^{g h} k^{h} R_{m n}^{h}\left(C_{m n k l}-\sigma_{m n} \delta_{k l}\right)\right]}
\end{aligned}
$$

\section{INTRAGRANULAR MODEL}

In the present model the hardening is described through several dislocation densities and their evolution. This microscopic model, based on experimental observations on BCC grains, is drawn from the works of Peeters [6]. It is assumed that the modelling at this scale captures the most relevant features of the dislocation structures, and therefore, the transient effects observed during changing strain paths can be reproduced adequately.

During plastic deformation, an intragranular microstructure develops, consisting of straight planar dislocations walls and of statistically stored dislocations in the cells. This microstructure is characterized by three dislocation densities (see Figure 1). The dislocations stored in the cells are represented by a single dislocation density for the whole slip systems. Two different dislocation densities are associated to six dislocation wall families: the density of immobile dislocations $\rho^{w d}$ stored in walls, and the polarity dislocation density $\rho^{w p}$. This last one is assumed to have a sign, and can be negative.

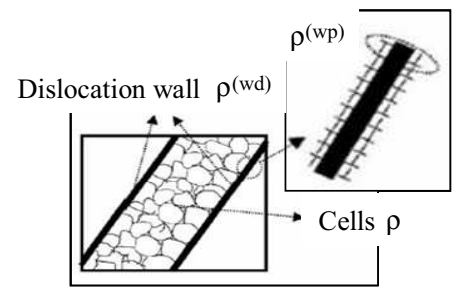

FIGURE 1. Schematic representation of intragranular microstructure.

The model produces at most two families of walls, in agreement with experimental observations; the primary family is generated parallel to the $\{110\}$-plane of the most active slip system, the second one is 
constructed in the same way, parallel to the $\{110\}$ plane of the second active slip system. The evolution of the three dislocation densities, introduced as internal state variables, are described as the separation into a storage term reflecting the immobilization mechanisms and a recovery term reflecting the annihilation mechanisms. The model distinguishes the evolution of walls created by the current slip processes from the evolution of walls formed by the prior slip activity.

The immobile dislocation density of each current wall $i$ can be calculated by:

$$
\dot{\rho}_{i}^{w d}=\frac{1}{b}\left(I^{w d} \sqrt{\rho_{i}^{w d}}-R^{w d} \rho_{i}^{w d}\right) \dot{\Gamma}_{i}
$$

where $\mathrm{b}$ is the magnitude of the Burgers vector, $\dot{\Gamma}_{i}$ is the total slip rate on the plane of the $i^{\text {th }}$ most active slip system. The immobilization coefficient $I^{w d}$ includes the probability of a mobile dislocation being trapped into a wall while the recovery coefficient $R^{w d}$ scales with the annihilation length.

The storage and recovery of polarity dislocations for each current wall $i$, can be described by:

$$
\dot{\rho}_{i}^{w p}=\left(\operatorname{sign}\left(\Phi_{i}^{w p}\right) I^{w p} \sqrt{\rho_{i}^{w d}+\left|\rho_{i}^{w p}\right|}-R^{w p} \rho_{i}^{w p}\right)\left|\Phi_{i}^{w p}\right|
$$

where $\Phi_{i}^{w p}=\sum_{s=1}^{n} \frac{\dot{\gamma}^{s}}{b} m^{s} \cdot n_{i}^{w}$ is the net flux of dislocations from slip systems non-coplanar of each current wall $i$, the scalar product of the unit slip direction vector $m^{s}$ of the system $s$ with the normal unit vector $n_{i}^{w}$ of the wall $i$ being equal to zero for slip activity coplanar with this wall. $I^{w p}$ and $R^{w p}$ are respectively the immobilization and the recovery coefficients of the polarity dislocations.

When a flux $\Phi_{i}^{w p}$, associated to a family $i$ of current walls, is reversed (for example during reverse tests), the polarity dislocations stuck at the border of theses walls are annihilated :

$$
\dot{\rho}_{i}^{w p}=-R_{r e v} \rho_{i}^{w p}\left|\Phi_{i}^{w p}\right|
$$

where $R_{r e v}$ is the recovery coefficient of the polarity dislocations responsible for the polarity of the walls due to a reversal of the net flux of dislocations.

A change in deformation path or a rotation of a crystal can lead to the activation of new slip systems. The mobile dislocations form new walls corresponding to the current deformation mode, but also disintegrate the old walls formed by prior slip activity, according to:

$$
\begin{aligned}
& \dot{\rho}_{i}^{w d}=-\frac{R_{n c g}}{b} \rho_{i}^{w d} \dot{\Gamma}_{n e w} \\
& \dot{\rho}_{i}^{w p}=-\frac{R_{n c g}}{b} \rho_{i}^{w p} \dot{\Gamma}_{n e w}
\end{aligned}
$$

where $\dot{\Gamma}_{n e w}$ denotes the total slip rate on the two crystallographic planes containing the highest slip activity and $R_{n c g}$ the annihilation coefficient of the latent walls.

During reverse tests, a change in deformation path leads to the activation of the same slip system but in the opposite sense. The polarity dislocations stuck at the border of the walls can move easily away and are annihilated by dislocations of opposite sign in cells. There is an increase of the annihilation rate for the randomly distributed cells:

$$
\dot{\rho}=\frac{1}{b}\left\langle(I \sqrt{\rho}-R \rho) \sum_{s=1}^{n}\left|\dot{\gamma}_{s}\right|-\Psi R_{2} \rho \frac{\rho_{\text {bausch }}}{2 \rho_{\text {sat }}^{w p}} \sum_{s=1}^{n}\left|\dot{\gamma}_{s}\right|\right\rangle
$$

where $I$ and $R$ represent respectively the immobilization and the recovery coefficients of the cells. If no fluxes are reversed $\Psi=0$, else $\Psi=1$ with $\rho_{\text {bausch }}=\left|\rho_{i}^{w p}\right|$ if only one flux, corresponding to the family $i$ of wall, is reversed, and $\rho_{\text {bausch }}=\sum_{i=1}^{2}\left|\rho_{i}^{w p}\right|$ if two fluxes are reversed.

The critical shear stress on slip system $s$ includes several contributions: $\tau_{0}$ represents all aspects of the microstructure that are not included in the internal variables (i.e. initial grain size), $\tau^{\text {cells }}$ depicts isotropic hardening due to the cells, $\tau^{w}$ introduces the latent hardening of the walls, and takes the contribution of polarity associated to the walls into account. The resultant critical shear stress is given by:

$$
\tau_{c}^{g}=\tau_{0}+(1-f) \tau^{\text {cells }}+f \sum_{i=1}^{6} \tau_{i g}^{w}
$$

with

$$
\begin{aligned}
\tau^{\text {cels }} & =\alpha G b \sqrt{\rho} \\
\tau_{i g}^{w} & =\alpha G b\left(\left\langle m_{s} \cdot n_{i}^{w} \operatorname{sign}\left(\rho_{i}^{w p}\right) \sqrt{\left|\rho_{i}^{w p}\right|}\right\rangle+\left|m_{s} \cdot n_{i}^{w}\right| \sqrt{\rho_{i}^{w d}}\right)
\end{aligned}
$$

where $\alpha$ represents the dislocation interaction parameter, $G$ the shear modulus and $f$ the volume fraction of walls. 


\section{SELF-CONSISTENT MODEL}

To deduce the polycrystalline behaviour from knowledge of the individual grains one, a selfconsistent scheme is used. Only main lines of this classical approach are presented here, the entire scheme details are developed in [4] and [7].

The macroscopic behaviour law linking the macroscopic nominal stress rate $\dot{N}$ to the macroscopic velocity gradient $G$ by the macroscopic tangent modulus $L$ has the same incremental form than the single crystal one:

$$
\dot{N}=L: G
$$

The macroscopic velocity gradient and the macroscopic nominal stress rate are given by the volume averages of the microscopic ones:

$$
G=\frac{1}{V} \int_{V} g d V, \quad \dot{N}=\frac{1}{V} \int_{V} \dot{n} d V
$$

To define the link existing between the macroscopic and microscopic quantities, fourth order concentration tensors $A$ and $B$ are introduced:

$$
g(x)=A(x): G, \quad \dot{n}(x)=B(x): \dot{N}
$$

By combining the local behaviour law with (16), (17) and (18) the macroscopic tangent modulus is given by:

$$
L=\overline{l(x): A(x)}
$$

It is assumed that the material is a polycrystal constituted of ellipsoidal grains with different crystallographic orientations. Behaviour and mechanical fields are considered homogeneous grain by grain. An indicator function $\theta^{I}$ for each grain $I$ of volume $V^{I}$ is defined by:

$$
\left\{\begin{array}{l}
\theta^{I}(x)=1 \text { if } x \in V^{I} \\
\theta^{I}(x)=0 \text { if } x \notin V^{I}
\end{array}\right.
$$

So:

$$
g(x)=\sum_{I=1}^{N \text { gran }} g^{I} \theta^{I}(x), \quad l(x)=\sum_{I=1}^{N \text { ggran }} l^{I} \theta^{I}(x)
$$

where $g^{I}$ (resp. $l^{I}$ ) is the volume average for the velocity gradient (resp. tangent modulus) of the grain $I$ and $N_{\text {gran }}$ is the number of grains composing the aggregate.

By introducing the Green's tensor, it can be demonstrated that the concentration tensor $A^{I}$ for the grain $I$ is given by:

$$
A^{I}=\left(I-T^{I I}:\left(l^{I}-L\right)\right)^{-1}: \overline{\left(I-T^{I I}:\left(l^{I}-L\right)\right)^{-1}}
$$

where $T^{I I}$ is the interaction tensor for the grain $I$, related to the Eshelby's tensor for an ellipsoidal inhomogeneity. In the case of a polycrystalline aggregate of $N_{\text {gran }}$ grains with their respective volume fraction $f^{I}$, the 1-site self-consistent expression corresponding to the self-consistent scheme in the sense of Hill can be obtained:

$$
L=\sum_{I=1}^{\text {Ngran }} f^{I} l^{I}: A^{I}
$$

\section{MATERIAL PARAMETERS IDENTIFICATION}

The adopted constitutive law parameters are presented in Table 1. They were obtained by fitting the simulated results with five different experimental tests:

- a monotonic shear with shear direction (SD) parallel to rolling direction (RD);

- two cross tests with different pre-strain of uniaxial traction in $\mathrm{RD}$, followed by simple shear with $\mathrm{SD}$ parallel to RD ;

- two reverse tests with different pre-strain of simple shear with $\mathrm{SD}$ parallel to $\mathrm{RD}$, then simple shear in the opposite sense.

The other standard constant material values (Burger's vector, shear modulus, the dislocation interaction parameter, dislocation walls volume fraction and initial critical shear stress on all slip systems) used in the model are reported in Table 2. The initial values of the dislocations densities are equal to $1.10^{9} \mathrm{~m}^{-2}$.

TABLE 1. Material parameters [6].

\begin{tabular}{ccccccccr}
\hline $\mathbf{I}$ & $\mathbf{R}[\mathbf{m}]$ & $\mathbf{I}^{\mathbf{w d}}$ & $\mathbf{R}^{\mathbf{w d}}[\mathbf{m}]$ & $\mathbf{R}_{\mathbf{n c g}}[\mathbf{m}]$ & $\mathbf{I}^{\mathbf{w p}}$ & $\mathbf{R}^{\mathbf{w p}}[\mathbf{m}]$ & $\mathbf{R}_{\text {rev }}[\mathbf{m}]$ & $\mathbf{R}_{\mathbf{2}}[\mathbf{m}]$ \\
\hline $2.210^{-2}$ & $8.510^{-10}$ & $9.410^{-1}$ & $2.610^{-8}$ & $2.310^{-9}$ & $5.010^{-2}$ & $3.810^{-9}$ & $1.010^{-8}$ & $1.010^{-8}$ \\
\hline
\end{tabular}

TABLE 2. Material constants [6].

\begin{tabular}{ccccc}
\hline $\mathbf{b}[\mathbf{m}]$ & $\mathbf{G}[\mathbf{M P a}]$ & $\boldsymbol{\alpha}$ & $\mathbf{f}$ & $\boldsymbol{\tau}_{\mathbf{0}}[\mathbf{M P a}]$ \\
\hline $2.4810^{-10}$ & $8.1610^{4}$ & 0.2 & 0.2 & 42.0 \\
\hline
\end{tabular}




\section{MICROSCOPIC VALIDATION}

In Figure 2 (left) a TEM micrograph in a grain of a specimen after a $15 \%$ shear test with SD parallel to RD is depicted. The traces of only one pronounced family of dislocation wall can be observed, parallel to the (101) slip plane. Figure 2 (right) shows a TEM micrograph in a grain of a specimen after a reverse test with SD parallel to RD. The traces of two pronounced families of dislocation walls can be observed, ones are parallel to the (011) slip plane and the others are parallel to the (10-1) slip plane.

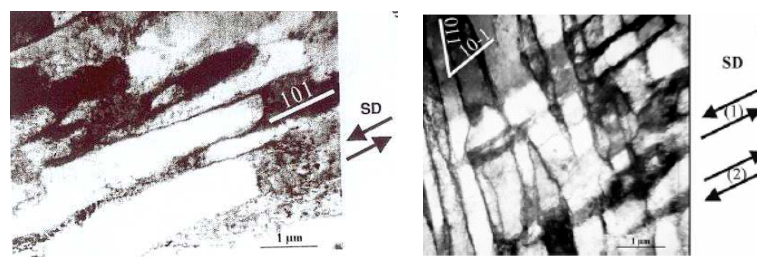

FIGURE 2. TEM micrographs in a grain with initial orientation: $\left(-27.2^{\circ}, 133.4^{\circ}, 53^{\circ}\right)$ after a shear test of $15 \%$ (left) ; $\left(43.3^{\circ}, 127.2^{\circ},-43.2^{\circ}\right)$ after a reverse test of $-30 \% / 30 \%$ (right) [6].

In Figures 3 and 4 , the dislocation density $\rho^{\text {wd }}$ stored in the walls (up) and the polarity dislocation density $\rho^{w p}$ associated to the walls (down), are depicted as function of the shear strain $\gamma$.

For the simple shear mode, there is only one pronounced family of walls, parallel to (101)-plane, predicted by the model, as it is shown on Figure 3 (up). This result is in agreement with the TEM observation. The polarity dislocation density is low (Figure 3 (down)) because there is only one pronounced activated slip system and the wall is generated parallel to the plane of this system.
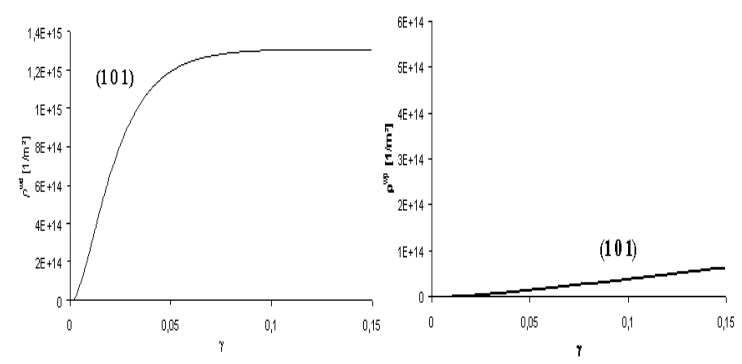

FIGURE 3. Evolution of the intensity and polarity of the dislocation walls in a crystal with initial orientation $\left(-27.2^{\circ}\right.$, $133.4^{\circ}, 53^{\circ}$ ) during a shear test.

For the reverse test, the model predicts the traces of both families of walls (Figure 4 (up)). The decrease of the dislocation density $\rho^{w d}$ at the beginning of the second loading, not present in the Peeters' simulation results [6], is due to the progressive activation of the slip systems. During the prestrain, the polarity along the walls is generated, due to the accumulation of dislocations of opposite sign at either side of the walls. During the reversal of the loading, the polarity dislocations are mobilized again and are annihilated with dislocations of opposite sign, leading to a depolarization of the walls. After this transition zone, the walls are polarized again, the polarity dislocations are accumulated at the borders of the current walls. These different steps are depicted on Figure 4 (down).
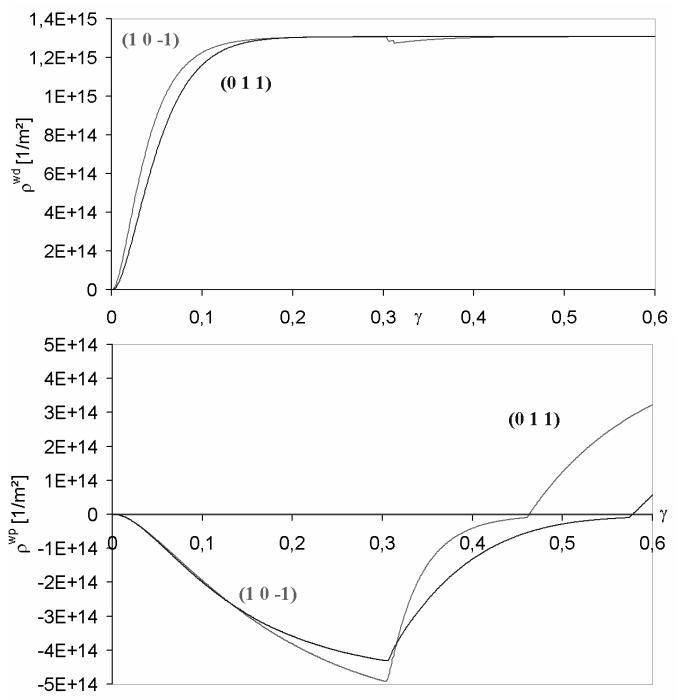

FIGURE 4. Evolution of the intensity and polarity of the dislocation walls in a crystal with initial orientation $\left(43.3^{\circ}\right.$, $127.2^{\circ},-43.2^{\circ}$ ) during a reverse test.

The grain rotation influences the macroscopic effects observed during strain path changes. A grain with stable initial orientation rotates very little during plastic strain while a grain with unstable initial orientation seeks to reach a stable orientation. Figure 5 clearly shows that the crystal with stable initial orientation is softer than the crystal with unstable initial orientation. The crystal with unstable initial orientation leads to a larger Bauschinger effect due to the higher density of polarity dislocations associated to the walls. 


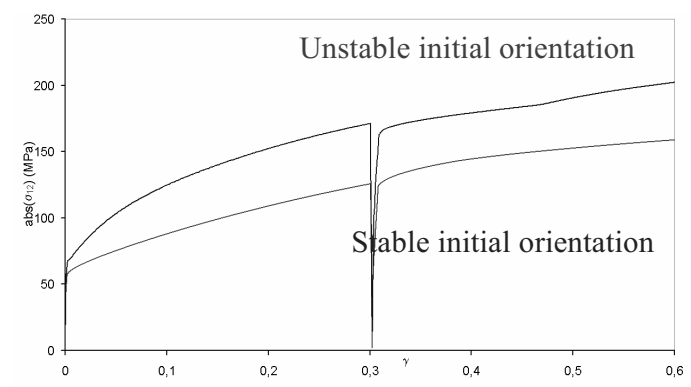

FIGURE 5. Stress-strain behaviour of two crystals with different initial orientations during a reverse test.

\section{MACROSCOPIC VALIDATION}

Figure 6 shows the stress-strain curves obtained by numerical simulations during reverse tests with different amounts of prestain, with the self-consistent model for a polycrystal of a ferritic steel.

During reverse tests, a drop in shear stress is obtained directly after reversal of the shear. This difference in plastic behaviour is known as the Bauschinger effect. The model correctly predicts this effect as shown in Figure 6. Moreover, reverse shearing leads to a strain-hardening stagnation, proportional to the amount of preshear. This plateau is also well described by the model.

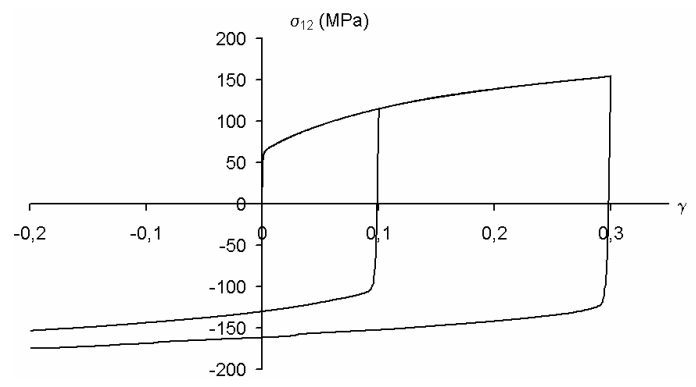

FIGURE 6. Simulation results for the stress-strain behaviour of an IF steel during monotonic and reverse tests.

Figure 7 shows the stress-strain curves obtained by numerical simulations during cross test with the selfconsistent model for a polycrystal of a ferritic steel.

During cross tests, immediately after the strainpath change, the flow stress is higher than that during monotonic deformation at the same accumulated strain. This is called the cross effect. The model correctly predicts this effect as shown in Figure 7.

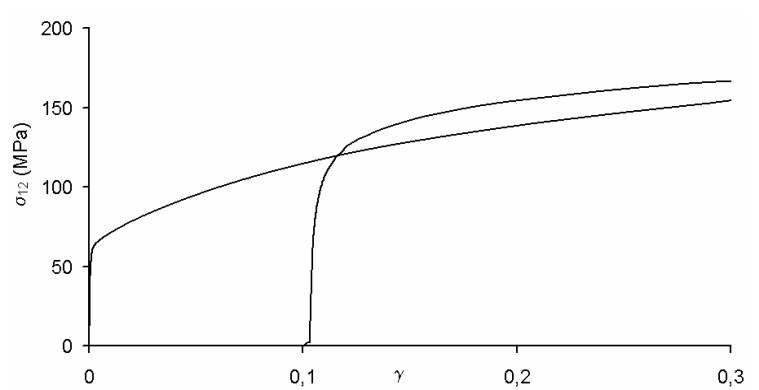

FIGURE 7. Simulation results for the stress-strain behaviour of an IF steel during monotonic and cross test.

\section{CONCLUSIONS}

It is shown that the model correctly reproduces the dislocation microstructure observed in TEM after different strain paths for crystal with different initial orientations. It is also shown that the initial orientation of the crystal has an influence on the stress-strain behaviour of the single crystal. The self-consistent model allows obtaining results at the microscropic scale in agreement with experimental observations.

This model is also able to reproduce macroscopic softening/hardening effects during strain path changes.

\section{REFERENCES}

1. R. J. Asaro, Journal of Applied Mechanics 50, pp. 921-934 (1983).

2. D. Peirce, Journal of the Mechanics and Physics Of Solids 2, pp. 133-153 (1983).

3. D. Pierce, R. J. Asaro and A. Needleman, Acta Metallurgica 31-12, pp. 1951-1976 (1983).

4. T. Iwakuma and S. Nemat-Nasser, "Finite ElasticPlastic Deformation Of Polycrystalline Metals And Composites", Technical report 83-3-51 (1983).

5. J. P. Lorrain, T. Ben Zineb, F. Abed-Meraim and M. Berveiller, International Journal of Forming Process 8-2, pp 135-158 (2005).

6. B. Peeters, "Multiscale Modelling Of The Induced Plastic Anisotropy In IF Steel During Sheet Forming", Ph.D. Thesis, Katholieke Universiteit Leuven (2002).

7. J. P. Lorrain, "Critère De Ductilité Basé Sur La Perte d'Ellipticité Du Module Tangent Elastoplastique Déduit d'Un Modèle Autocohérent", Ph.D. Thesis, ENSAM Metz (2005). 